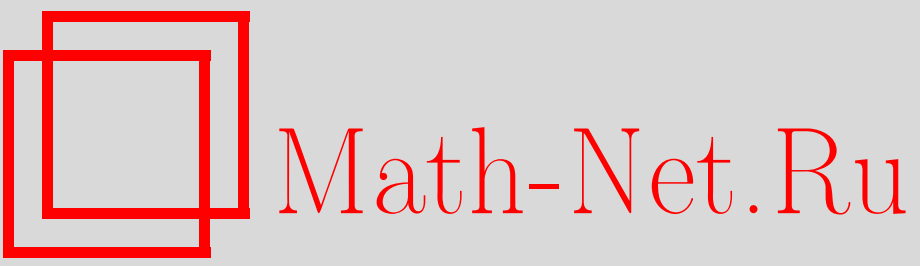

О. И. Завьялов, А. М. Малокостов, Функция Вигнера для свободных релятивистских частиц, ТМФ, 1999, том 119, номер 1, 67-72

DOI: https://doi.org/10.4213/tmf728

Использование Общероссийского математического портала Math-Net.Ru подразумевает, что вы прочитали и согласны с пользовательским соглашением

http://www.mathnet.ru/rus/agreement

Параметры загрузки:

IP: 35.174 .16 .151

26 апреля 2023 г., 10:31:25 


\section{ФУНКЦИЯ ВИГНЕРА ДЛЯ СВОБОДНЫХ РЕЛЯТИВИСТСКИХ ЧАСТИЦ}

Представлено обобщение функции Вигнера на случай свободной частицы с "релятивистским" гамильтонианом $H(\mathbf{p})=\sqrt{\mathbf{p}^{2}+m^{2}}$.

\section{1. ВВЕДЕНИЕ. НЕРЕЛЯТИВИСТСКИЙ СЛУЧАЙ}

Известно [1], что квантовое поведение нерелятивистской частицы, волновая функция которой в импульсном представлении (в момент времени $t=0)$ есть $\psi(\mathbf{p})$, может быть описано в эквивалентной “классической” форме с помошью функции Вигнера $W(\mathbf{p}, \mathbf{x}, t)$ :

$$
\begin{aligned}
W(\mathbf{p}, \mathbf{x}, t)= & \frac{1}{(2 \pi)^{3}} \int d \mathbf{p}_{1} d \mathbf{p}_{2} \psi^{*}\left(\mathbf{p}_{1}\right) \psi\left(\mathbf{p}_{2}\right) \delta\left(\mathbf{p}-\frac{\mathbf{p}_{1}+\mathbf{p}_{2}}{2}\right) \times \\
& \times \exp \left\{i\left(\frac{\mathbf{p}_{1}^{2}}{2 m}-\frac{\mathbf{p}_{2}^{2}}{2 m}\right) t+i\left(\mathbf{p}_{2}-\mathbf{p}_{1}\right) \mathbf{x}\right\}
\end{aligned}
$$

Эту величину можно интерпретировать (см. обзор [2]) как плотность распределения в шестимерном фазовом пространстве $\{\mathbf{p}, \mathbf{x}\}$ ансамбля свободных классических нерелятивистских частиц, соответствующих гамильтониану

$$
H=\frac{p^{2}}{2 m} \text {. }
$$

Легко проверить, что средние значения $\overline{f(\mathbf{x})}$ и $\overline{\varphi(\mathbf{p})}$ функций $f(\mathbf{x})$ и $\varphi(\mathbf{p})$ по этому классическому ансамблю совпадают с соответствуюшими квантовыми матричными элементами:

$$
\begin{aligned}
& \overline{f(\mathbf{x})}=\int d \mathbf{p} d \mathbf{x} W(\mathbf{p}, \mathbf{x}, t) f(\mathbf{x})=\int d \mathbf{x} \widetilde{\psi}^{*}(\mathbf{x}, t) f(\widehat{\mathbf{x}}) \widetilde{\psi}(\mathbf{x}, t) \\
& \overline{\varphi(\mathbf{p})}=\int d \mathbf{p} d \mathbf{x} W(\mathbf{p}, \mathbf{x}, t) \varphi(\mathbf{p})=\int d \mathbf{p} \psi^{*}(\mathbf{p}, t) \varphi(\widehat{\mathbf{p}}) \psi(\mathbf{p}, t)
\end{aligned}
$$

Здесь

$$
\widetilde{\psi}(\mathbf{x}, t)=\frac{1}{(2 \pi)^{3 / 2}} \int d \mathbf{p} e^{i \mathbf{p} \mathbf{x}} \psi(\mathbf{p}, t)
$$

* Математический институт им. В. А. Стеклова РАН, Москва, Россия 
и

$$
\psi(\mathbf{p}, t)=e^{-i \frac{k^{2}}{2 m} t} \psi(\mathbf{p})
$$

- волновые функции в координатном и импульсном представлениях, соответственно, в момент времени $t$. Более того, среднее значение функции $F(\mathbf{p}, \mathbf{x})$ по этому классическому ансамблю совпадает с матричным элементом

$$
\langle\widehat{F}(\widehat{\mathbf{p}}, \widehat{\mathbf{x}})\rangle,
$$

где $\widehat{F}$ - вейлевское квантование классической функции $F$.

Таким образом, идея Вигнера состоит в том, что квантовая эволюция и эволюция классического ансамбля (1) тождественны. Однако эту тождественность не следует понимать слишком буквально: функция Вигнера не обязательно положительна.

Любопытно, что обобшение соотношения (1) на релятивистский случай, т.е. для гамильтониана

$$
H(\mathbf{p})=\omega(\mathbf{p})=\sqrt{\mathbf{p}^{2}+m^{2}},
$$

по-видимому, неизвестно. В настоящей работе мы представим это обобщение.

Перечислим главные свойства функции Вигнера (1):

1) она билинейна по волновой функции $\psi(\mathbf{p})$;

2 ) после интегрирования функции $W$ по координате $\mathbf{x}$ получается плотность вероятности в импульсном пространстве

$$
\psi^{*}(\mathbf{p}, t) \psi(\mathbf{p}, t)=\int d \mathbf{x} W(\mathbf{p}, \mathbf{x}, t) ;
$$

3) после интегрирования функции $W$ по импульсу р получается плотность вероятности в координатном пространстве

$$
\widetilde{\psi}^{*}(\mathbf{x}, t) \widetilde{\psi}(\mathbf{x}, t)=\int d \mathbf{p} W(\mathbf{p}, \mathbf{x}, t) ;
$$

4) функция Вигнера допускает классический закон эволюции, а именно:

$$
W(\mathbf{p}, \mathbf{x}, t+\tau)=W\left(\mathbf{p}, \mathbf{x}-\frac{\mathbf{p}}{m} \tau, t\right) .
$$

Свойства 1-4 легко проверить, однако мы отложим соответствующие вычисления, поскольку эти формулы будут следовать из релятивистских соотношений в пределе малых импульсов.

Хорошо известно, что наивная квантово-механическая схема несовместима с релятивистскими принципами (это было фактически главной причиной создания квантовой теории поля). Например, трудности возникают уже при построении самого оператора координаты $\widehat{\mathbf{x}}[3]$. Тем не менее предположим, что свободная частица с гамильтонианом (5) обладает волновой функцией в импульсном представлении (функцией на верхней поле массового гиперболоида), равной $\psi(\mathbf{p})$. Скалярное произведение между двумя такими функциями $\psi_{1}(\mathbf{p})$ и $\psi_{2}(\mathbf{p})$ есть

$$
\left\langle\psi_{1}(\mathbf{p}), \psi_{2}(\mathbf{p})\right\rangle=\int d \mu(\mathbf{p}) \psi_{1}^{*}(\mathbf{p}) \psi_{2}(\mathbf{p}),
$$


где

$$
d \mu(\mathbf{p})=\frac{d \mathbf{p}}{\omega(\mathbf{p})}
$$

- инвариантная мера на массовом гиперболоиде. Считается, что трансформационные свойства импульсных волновых функций $\psi(\mathbf{p})$ при преобразованиях Лоренца $\Lambda$ таковы:

$$
\psi_{\Lambda}(\mathbf{p})=\psi(\mathbf{\Lambda} \mathbf{p}) .
$$

Естественно перейти в импульсное пространство $L_{2}\left(d \mathbf{p}, \mathbb{R}^{3}\right)$ волновых функций $\psi^{\prime}(\mathbf{p})$ с лебеговой мерой $d \mathbf{p}$. Соответствуюший изоморфизм дается формулой

$$
\psi^{\prime}(\mathbf{p})=\frac{\psi(\mathbf{p})}{\sqrt{\omega(\mathbf{p})}},
$$

а общий закон преобразования волновой функции при преобразовании Лоренца может принимать форму

$$
\psi_{\Lambda}^{\prime}(\mathbf{p})=\sqrt{\frac{\omega(\boldsymbol{\Lambda} \mathbf{p})}{\omega(\mathbf{p})}} e^{i \Omega(\Lambda \mathbf{p})-i \Omega(\mathbf{p})} \psi^{\prime}(\boldsymbol{\Lambda} \mathbf{p}) .
$$

Здесь $\Omega(\mathbf{p})$ - произвольная вешественная функция.

Оператор импульса $\widehat{\mathbf{p}}$ в этом пространстве - просто оператор умножения:

$$
\widehat{\mathbf{p}} \psi^{\prime}(\mathbf{p})=\mathbf{p} \psi^{\prime}(\mathbf{p}) .
$$

Из канонических коммутационных соотношений следует, что оператор координаты $\widehat{\mathbf{x}}$ (если таковой существует) может быть выбран в виде

$$
\widehat{\mathbf{x}}=i \frac{\partial}{\partial \mathbf{p}} \text {. }
$$

Соответственно координатная волновая функция $\widetilde{\psi^{\prime}}(\mathbf{x})$ (если таковая существует) является просто преобразованием Фурье импульсной волновой функции $\psi^{\prime}(\mathbf{p})$ :

$$
\widetilde{\psi^{\prime}}(\mathbf{x}, t)=\frac{1}{(2 \pi)^{3 / 2}} \int d \mathbf{p} e^{i \mathbf{p x}} \psi^{\prime}(\mathbf{p}) e^{-i \omega(\mathbf{p}) t} .
$$

\section{2. РЕЛЯТИВИСТСКАЯ ФУНКЦИЯ ВИГНЕРА}

Перейдем теперь к рассмотрению релятивистского случая.

Требуется найти функцию $W(\mathbf{p}, \mathbf{x}, t)$, имеюшую следуюшие свойства (унаследованные от нерелятивистского случая):

1) она билинейна по функциям $\psi^{\prime}(\mathbf{p})$;

2 ) будучи проинтегрированной по $\mathbf{x}$, функция $W$ дает плотность вероятности в импульсном пространстве

$$
\psi^{\prime *}(\mathbf{p}, t) \psi^{\prime}(\mathbf{p}, t)=\int d \mathbf{x} W(\mathbf{p}, \mathbf{x}, t)
$$


3) будучи проинтегрированной по $\mathbf{p}$, функция $W$ дает плотность вероятности в координатном пространстве

$$
\widetilde{\psi^{\prime}}(\mathbf{p}, t) \widetilde{\psi^{\prime}}(\mathbf{x}, t)=\int d \mathbf{p} W(\mathbf{p}, \mathbf{x}, t)
$$

4) функция Вигнера имеет классический закон эволюции, а именно:

$$
W(\mathbf{p}, \mathbf{x}, t+\tau)=W\left(\mathbf{p}, \mathbf{x}-\frac{\mathbf{p}}{\omega(\mathbf{p})} \tau, t\right)
$$

$5)$ в пределе малых импульсов функция $W$ переходит в нерелятивистскую функцию Вигнера, определенную равенством (1).

Решение этой задачи дается формулой

$$
\begin{aligned}
W(\mathbf{p}, \mathbf{x}, t)= & \frac{1}{(2 \pi)^{3}} \int d \mathbf{p}_{1} d \mathbf{p}_{2} \psi^{\prime *}\left(\mathbf{p}_{1}\right) \psi^{\prime}\left(\mathbf{p}_{2}\right) \delta\left(\mathbf{p}-\left(\mathbf{p}_{1} \dot{+} \mathbf{p}_{2}\right)\right) \times \\
& \times \exp \left(i\left(\omega\left(\mathbf{p}_{1}\right)-\omega\left(\mathbf{p}_{2}\right)\right) t+i\left(\mathbf{p}_{2}-\mathbf{p}_{1}\right) \mathbf{x}\right)
\end{aligned}
$$

Здесь символ + обозначает специальную "сумму на массовом гиперболоиде" : если ввести 4-векторы $P_{1}=\left(\omega\left(\mathbf{p}_{1}\right), \mathbf{p}_{1}\right)$ и $P_{2}=\left(\omega\left(\mathbf{p}_{2}\right), \mathbf{p}_{2}\right)$, то по определению

$$
P_{1}+P_{2} \equiv m \frac{P_{1}+P_{2}}{\sqrt{\left(P_{1}+P_{2}\right)^{2}}},
$$

a $\mathbf{p}_{1} \dot{+} \mathbf{p}_{2}-$ просто пространственная часть 4 -вектора $P_{1} \dot{+} P_{2}$.

Другими словами,

$$
\mathbf{p}_{1} \dot{+} \mathbf{p}_{2}=m \frac{\mathbf{p}_{1}+\mathbf{p}_{2}}{\sqrt{2\left(m^{2}+\omega\left(\mathbf{p}_{1}\right) \omega\left(\mathbf{p}_{2}\right)-\mathbf{p}_{1} \mathbf{p}_{2}\right)}}
$$

Доказательство свойств 1-5 будет дано в следуюшем разделе.

Разумеется, ситуация с физической интерпретацией релятивистской функции $W$ нисколько не лучше, чем в нерелятивистском случае. Она также может быть отрицательной. Далее, средние значения по классическому “ансамблю" для "смешанных" функций (зависящих одновременно от координат и импульсов) не имеют ничего общего с квантовыми матричными элементами, по крайней мере в рамках вейлевского квантования. Более того, из обших принципов классической механики следует, что функция $W(\mathbf{p}, \mathbf{x}, t)$ должна быть скаляром по отношению к преобразованиям Лоренца, что никоим образом несовместимо с законами преобразования волновых функций (6). Это показывает, что даже для случая свободных частиц несовместимость между принципами квантовой механики и специальной теории относительности, возможно, является более глубокой, чем принято думать. Однако мы надеемся, что функция (8) все же может быть полезна, например, в теории "непрерывных измерений". Нам она интересна для анализа специального представления канонических коммутационных соотношений, которое мы пытаемся применить к квантовой теории поля [4]. 


\section{3. ДОКАЗАТЕЛЬСТВО СВОЙСТВ 1-5}

Свойство 1 очевидно. Свойство 5 может быть получено из (9) в пределе $\omega \rightarrow m$. Свойство 3 может быть проверено непосредственно. В самом деле, интегрирование функции $W$ по импульсу $\mathbf{p}$ снимается $\delta$-функцией. В результате остается как раз плотность вероятности $\widetilde{\psi^{\prime}}{ }^{*}(\mathbf{x}, t) \widetilde{\psi^{\prime}}(\mathbf{x}, t)$. Рассуждения, приводящие к свойству 2, также очень просты: в результате интегрирования по координате $\mathbf{x}$ появляется $\delta$-функция:

$$
\frac{1}{(2 \pi)^{3}} \int d \mathbf{x} \exp \left\{i \mathbf{x}\left(\mathbf{p}_{1}-\mathbf{p}_{2}\right)\right\}=\delta\left(\mathbf{p}_{1}-\mathbf{p}_{2}\right),
$$

так что эффективно

$$
\mathbf{p}_{2}=\mathbf{p}_{1}
$$

Таким образом, первоначальная $\delta$-функция преврашается в $\delta\left(\mathbf{p}-\mathbf{p}_{1}\right)$, а интегрирование по $\mathbf{p}_{1}$ немедленно приводит к нужному соотношению свойства 2.

Прежде чем обратиться к свойству 4, отметим, что выполняется следующее равенство: если $\mathbf{p}=\mathbf{p}_{1}+\mathbf{p}_{2}$, то

$$
\omega(\mathbf{p})=\frac{m}{\sqrt{2}} \frac{\omega\left(\mathbf{p}_{1}\right)+\omega\left(\mathbf{p}_{2}\right)}{\sqrt{m^{2}+\omega\left(\mathbf{p}_{1}\right) \omega\left(\mathbf{p}_{2}\right)-\mathbf{p}_{1} \mathbf{p}_{2}}} .
$$

Теперь, чтобы доказать свойство 4 (учитывая формулу (10)), достаточно убедиться в том, что

$$
-\tau\left(\omega\left(\mathbf{p}_{2}\right)-\omega\left(\mathbf{p}_{1}\right)\right)+\left(\mathbf{p}_{1}+\mathbf{p}_{2}\right) \frac{\mathbf{p} \tau}{\omega(\mathbf{p})}=0 .
$$

Следуюшая цепочка преобразований делает это очевидным:

$$
\begin{aligned}
\omega\left(\mathbf{p}_{2}\right)-\omega\left(\mathbf{p}_{1}\right)= & \frac{\left(\omega\left(\mathbf{p}_{2}\right)\right)^{2}-\left(\omega\left(\mathbf{p}_{1}\right)\right)^{2}}{\omega\left(\mathbf{p}_{2}\right)+\omega\left(\mathbf{p}_{1}\right)}=\frac{\mathbf{p}_{2}^{2}-\mathbf{p}_{1}^{2}}{\omega\left(\mathbf{p}_{2}\right)+\omega\left(\mathbf{p}_{1}\right)}= \\
= & \frac{\left(\mathbf{p}_{2}-\mathbf{p}_{1}\right)\left(\mathbf{p}_{2}+\mathbf{p}_{1}\right)}{\sqrt{m^{2}+\omega\left(\mathbf{p}_{1}\right) \omega\left(\mathbf{p}_{2}\right)-\mathbf{p}_{1} \mathbf{p}_{2}}} \times \\
& \times \frac{\sqrt{m^{2}+\omega\left(\mathbf{p}_{1}\right) \omega\left(\mathbf{p}_{2}\right)-\mathbf{p}_{1} \mathbf{p}_{2}}}{\omega\left(\mathbf{p}_{1}\right)+\omega\left(\mathbf{p}_{2}\right)} .
\end{aligned}
$$

Иногда удобно рассматривать модельный случай двумерного пространства-времени. В этом случае импульсное пространство, соответствуюшее массовой оболочке $P^{2}=m^{2}$, будет одномерным:

$$
P=\{m \operatorname{ch} \gamma, m \operatorname{sh} \gamma\} .
$$

Тогда $\dot{+}-\delta$-функция может быть эффективно проинтегрирована. При этом для функции Вигнера получаем

$$
\begin{aligned}
W(p, x, t)= & \frac{2 m^{3}}{\operatorname{ch} \Gamma} \int_{-\infty}^{+\infty} d \beta \operatorname{ch}(\Gamma+\beta) \operatorname{ch}(\Gamma-\beta) \times \\
& \times \psi^{\prime *}(m \operatorname{sh}(\Gamma+\beta)) \psi^{\prime}(m \operatorname{sh}(\Gamma-\beta)) \times \\
& \times \exp \left\{\frac{i t}{m}[\operatorname{ch}(\Gamma+\beta)-\operatorname{ch}(\Gamma-\beta)]-\frac{i x}{m}[\operatorname{sh}(\Gamma+\beta)-\operatorname{sh}(\Gamma-\beta)]\right\} .
\end{aligned}
$$


В самом деле, перейдем в (8) к аналогичным переменным для одномерных импульсов $p_{1}$ и $p_{2}$ :

$$
\begin{aligned}
\frac{p_{1}}{m} & =\operatorname{sh} \gamma_{1}, & \frac{p_{2}}{m} & =\operatorname{sh} \gamma_{2}, \\
\frac{d p_{1}}{m} & =\operatorname{ch} \gamma_{1} d \gamma_{1}, & \frac{d p_{2}}{m} & =\operatorname{ch} \gamma_{2} d \gamma_{2} .
\end{aligned}
$$

После этого функция Вигнера $W$ принимает форму

$$
\begin{aligned}
W= & m^{2} \int d \gamma_{1} d \gamma_{2} \operatorname{ch} \gamma_{1} \operatorname{ch} \gamma_{2} \psi^{\prime *}\left(m \operatorname{sh} \gamma_{1}\right) \psi^{\prime}\left(m \operatorname{sh} \gamma_{2}\right) \times \\
& \times \exp \left\{i \frac{t}{m}\left(\operatorname{ch} \gamma_{1}-\operatorname{ch} \gamma_{2}\right)-i \frac{x}{m}\left(\operatorname{sh} \gamma_{1}-\operatorname{sh} \gamma_{2}\right)\right\} \times \\
& \times m \delta\left(\operatorname{sh} \Gamma-\frac{\operatorname{sh} \gamma_{1}+\operatorname{sh} \gamma_{2}}{\sqrt{2} \sqrt{1+\operatorname{ch} \gamma_{1} \operatorname{ch~} \gamma_{2}-\operatorname{sh} \gamma_{1} \operatorname{sh} \gamma_{2}}}\right)
\end{aligned}
$$

Выражение под знаком квадратного корня в аргументе $\delta$-функции можно преобразовать следующим образом:

$$
\begin{aligned}
& 1+\operatorname{ch} \gamma_{1} \operatorname{ch} \gamma_{2}-\operatorname{sh} \gamma_{1} \operatorname{sh} \gamma_{2}=1+\operatorname{ch}\left(\gamma_{1}-\gamma_{2}\right)= \\
& =1+\operatorname{ch}^{2}\left(\frac{\gamma_{1}-\gamma_{2}}{2}\right)+\operatorname{sh}^{2}\left(\frac{\gamma_{1}-\gamma_{2}}{2}\right)=2 \operatorname{ch}^{2}\left(\frac{\gamma_{1}-\gamma_{2}}{2}\right) .
\end{aligned}
$$

Таким образом, полный интеграл (13) преобразуется к виду

где

$$
\begin{aligned}
W & =m^{3} \int d \gamma_{1} d \gamma_{2} \Psi^{*}\left(\gamma_{1}, x, t\right) \Psi\left(\gamma_{2}, x, t\right) \delta\left(\operatorname{sh} \Gamma-\operatorname{sh} \frac{\gamma_{1}+\gamma_{2}}{2}\right) \operatorname{ch} \gamma_{1} \operatorname{ch} \gamma_{2}= \\
& =m^{3} \int d \gamma_{1} d \gamma_{2} \Psi^{*}\left(\gamma_{1}, x, t\right) \Psi\left(\gamma_{2}, x, t\right) \frac{1}{\operatorname{ch} \Gamma} \delta\left(\Gamma-\frac{\gamma_{1}+\gamma_{2}}{2}\right) \operatorname{ch} \gamma_{1} \operatorname{ch} \gamma_{2}
\end{aligned}
$$

$$
\Psi(\gamma, x, t)=\psi^{\prime}(m \operatorname{sh} \gamma) \exp \left(-i \frac{t}{m} \operatorname{ch} \gamma+i \frac{x}{m} \operatorname{sh} \gamma\right) .
$$

Перейдем к новым переменным $\alpha$ и $\beta$ :

$$
\gamma_{1}=\alpha+\beta, \quad \gamma_{2}=\alpha-\beta .
$$

В результате интегрирование по $\alpha$ снимается $\delta$-функцией, и мы приходим к равенству (12).

В трехмерном случае эффективное интегрирование с помощью $\delta$-функции также может быть произведено.

Благодарности. Настоящая работа поддержана Российским фондом фундаментальных исследований, грант 98-01-00162а.

\section{Список литературы}

[1] E. P. Wigner. Phys. Rev. 1932. V. 40. P. 749.

[2] В. И. Татарский. УФН. 1983. Т. 139. С. 587.

[3] T. D. Newton, E. P. Wigner. Rev. Mod. Phys. 1949. V. 21. P. 400.

[4] О.И. Завьялов, А. М. Малокостов. Квантовая теория поля с нефоковскими асимптотическими полями: существование $S$-матрицы. ТМФ (в печати).

Поступила в редакцию 17.IX.1998 г. 